\title{
Facile Denitrosation of cyclic $N$-Nitrosamines with Hydrazoic acid
}

\author{
S. Ponnuswamy*, A. Akila, D. Kiruthiga devi \\ P. G. \& Research Department of Chemistry, Government Arts College (Autonomous), Coimbatore 641 018, Tamil Nadu, India. \\ * corresponding author. E-mail address : kspons2001@.gmail.com
}

\section{Supplementary Information}

\section{3,3-Dimethyl-2,7-diphenyl-1,4-diazepan-5-one (1)}

In a typical reaction dry, powdered 3,3-dimethyl-2,6-diphenylpiperidin-4-one hydrochloride $(10 \mathrm{~g}, 31.70 \mathrm{mmol})$ was added, in portions, to cold Con. $\mathrm{H}_{2} \mathrm{SO}_{4}(25 \mathrm{ml})$ in a conical flask equipped with a magnetic stirrer. After the dissolution was complete, the temperature of the solution was allowed to rise to $25^{\circ} \mathrm{C}$. Then, $\mathrm{NaN}_{3}(3 \mathrm{~g}, 46.15 \mathrm{mmol})$ was added in portions of $0.1 \mathrm{~g}$, with vigorous stirring. After the addition was over, the solution was poured into crushed ice and stirred well with a glass rod and cold $\mathrm{NaOH}$ solution $(2 \mathrm{~N})$ was added slowly with stirring until the $\mathrm{pH}$ was 8 . A white solid separated out. After keeping the mixture at room temperature for overnight the solid was separated by filtration through a Buchner funnel, washed free of $\mathrm{NaOH}$ and dried. The dried solid was dissolved in benzene and filtered through a fluted filter paper and the solution was concentrated. The solution was kept aside for crystallization. The crystals obtained were separated and then recrystallized from ethanol and pet ether $\left(60-80^{\circ} \mathrm{C}\right)$. yield: $49.46 \%$, mp $210-211^{\circ} \mathrm{C} ; 209-210^{\circ} \mathrm{C} .^{[9]}$

The compounds 9-13 have been prepared similarly. ${ }^{[5]}$

\section{2,6-Diphenylpiperidin-4-one (14)}


Ammonium acetate $(100 \mathrm{mmol})$, benzaldehyde $(200 \mathrm{mmol})$ and acetone $(200 \mathrm{mmol})$ were dissolved in ethanol $(20 \mathrm{ml})$ and the resulting solution was heated on a hot plate with gentle swirling until the colour of the mixture changed to orange. The mixture was cooled under running tap water and poured into ether $(100 \mathrm{ml})$. The ether insoluble 2,4,6,8-tetraphenyl-3,7diazabicyclo[3.3.1]nonan-9-one was filtered off and conc. $\mathrm{HCl}(14 \mathrm{ml})$ was added to the filtrate. The precipitated $r$-2,c-6-diphenylpiperidin-4-one hydrochloride was collected by filtration and recrystallised from ethanol-ether by dissolving it in the minimum amount of ethanol and then adding dry ether until turbidity appeared in the cold solution. The hydrochloride was dispersed in acetone $(50 \mathrm{ml})$ and concentrated aqueous ammonia was added drop wise until a clear solution was obtained. The solution was poured into cold water $(500 \mathrm{ml})$ and the precipitated solid was collected and recrystallized form ethanol. mp: $101-102^{\circ} \mathrm{C} ; 102-103^{\circ} \mathrm{C}^{[6]}$

\section{3-Methyl-2,6-diphenylpiperidin-4-one (15)}

Ammonium acetate $(100 \mathrm{mmol})$ was dissolved in ethanol $(80 \mathrm{ml})$ by heating. Benzaldehyde $(200 \mathrm{mmol})$ and butan-2-one $(100 \mathrm{mmol})$ were added to this solution and the mixture was heated until the colour of the solution changed to yellow. The solution was stored at room temperature for about 5 hours. The solid that precipitated was filtered off, washed and recrystallised from ethanol. $\mathrm{mp}: 86-87^{\circ} \mathrm{C} ; 85-86^{\circ} \mathrm{C} .^{[8]}$

The compounds 16-19 have been prepared by following the similar procedure. ${ }^{[6-8]}$

\section{2,6-Diphenylpiperidine (26)}


A mixture of piperidin-4-one $(10 \mathrm{~m} . \mathrm{mol})$, and $80 \%$ hydrazine hydrate $(3.1 \mathrm{ml}$.$) in$ diethylene glycol $(100 \mathrm{ml})$ was heated on a steam bath for 2 hours. Potassium hydroxide pellets $(2.8 \mathrm{~g})$ were added to the mixture and the contents were refluxed vigorously on a heating mantle for another 2 hours and the reaction mixture was cooled. The product formed was filtered and recrystallised from ethanol. Melting point: $69-70^{\circ} \mathrm{C} ; 69-71{ }^{\circ} \mathrm{C} .{ }^{[6]}$

The compounds $\mathbf{2 7 - 3 1}$ have been prepared by following the similar procedure. ${ }^{[6]}$ 

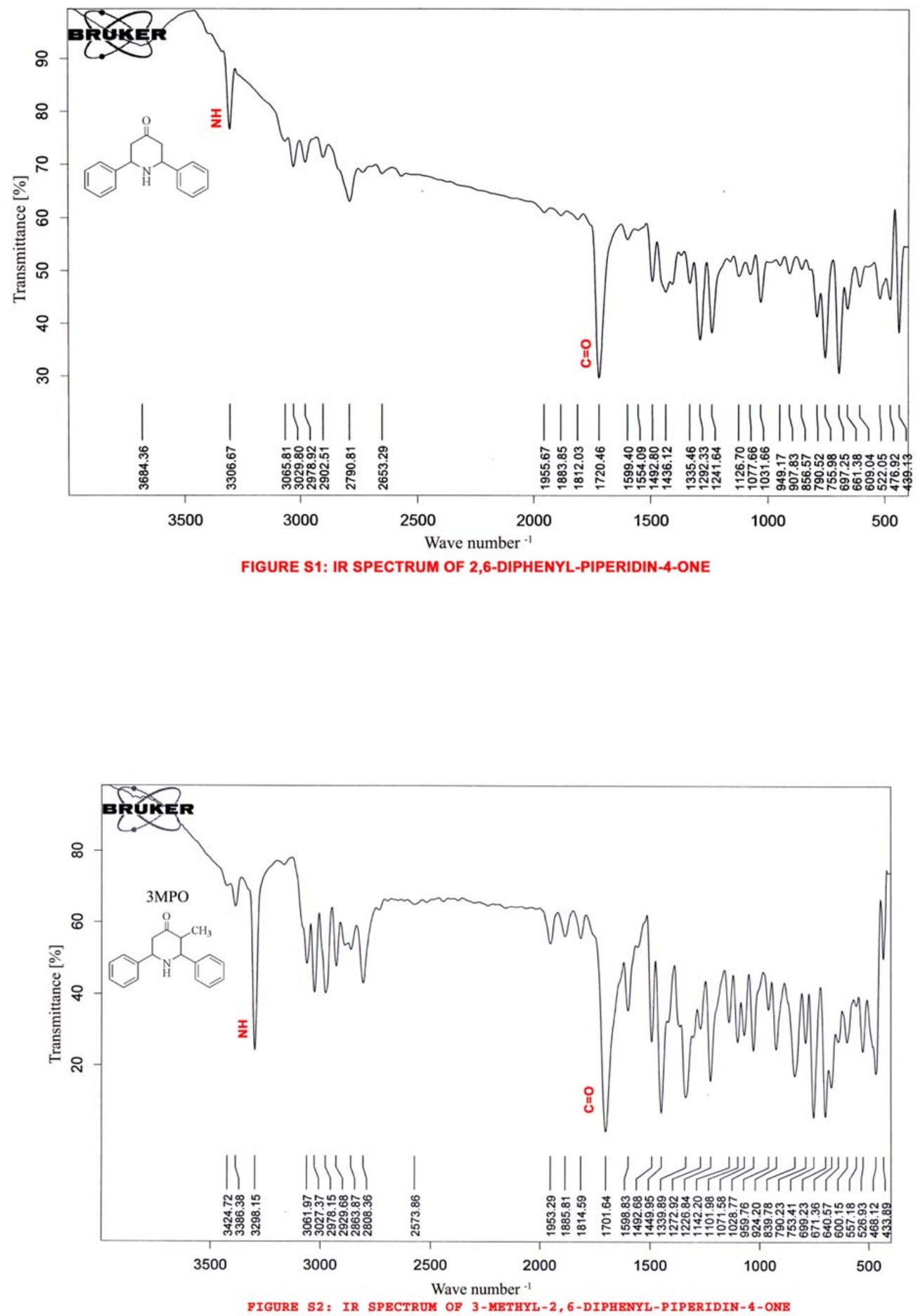

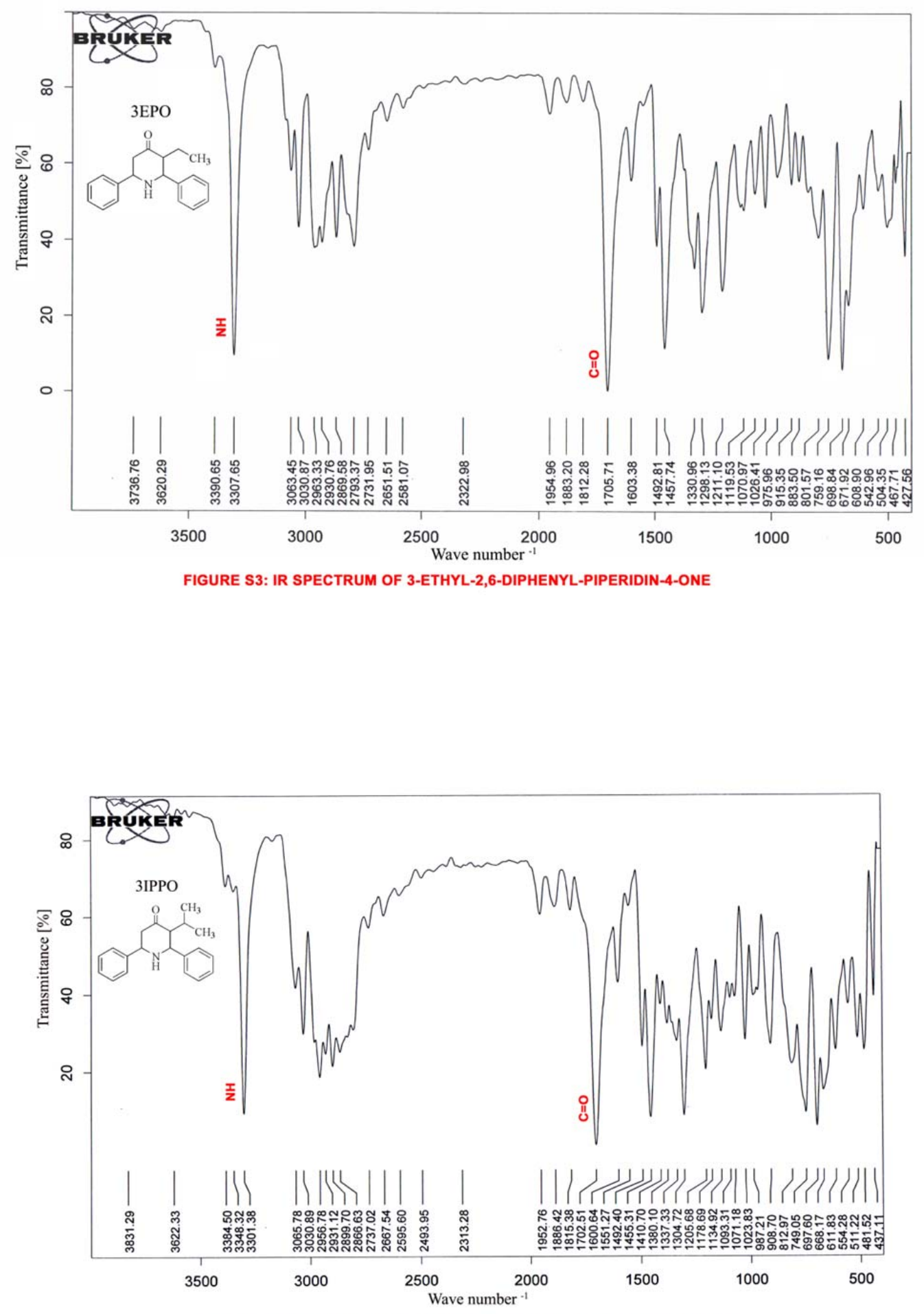

FIGURE S4: IR SPECTRUM OF 3-ISOPROPYL-2,6-DIPHENYL-PIPERIDIN-4-ONE 

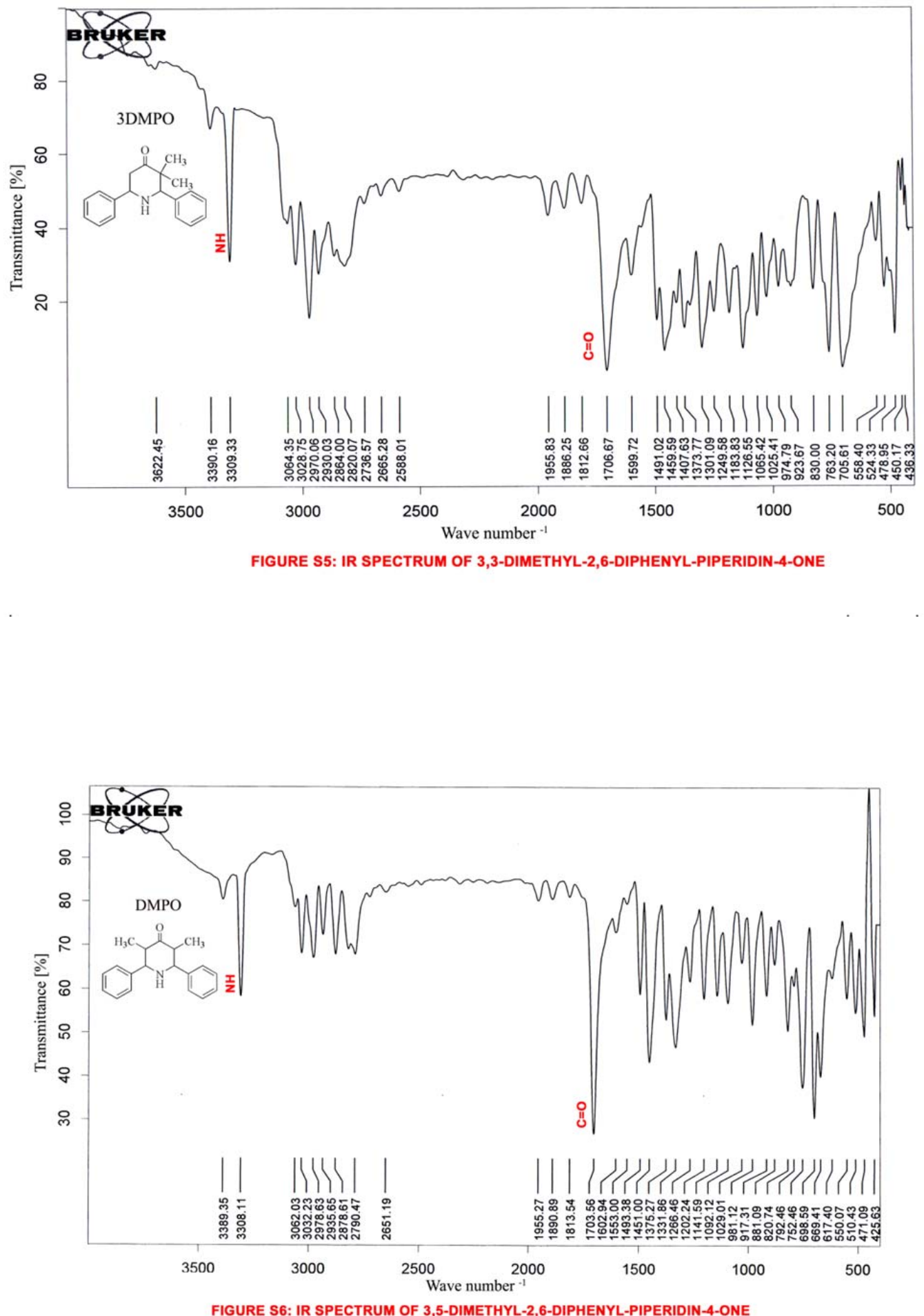


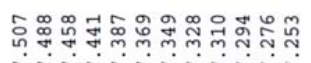
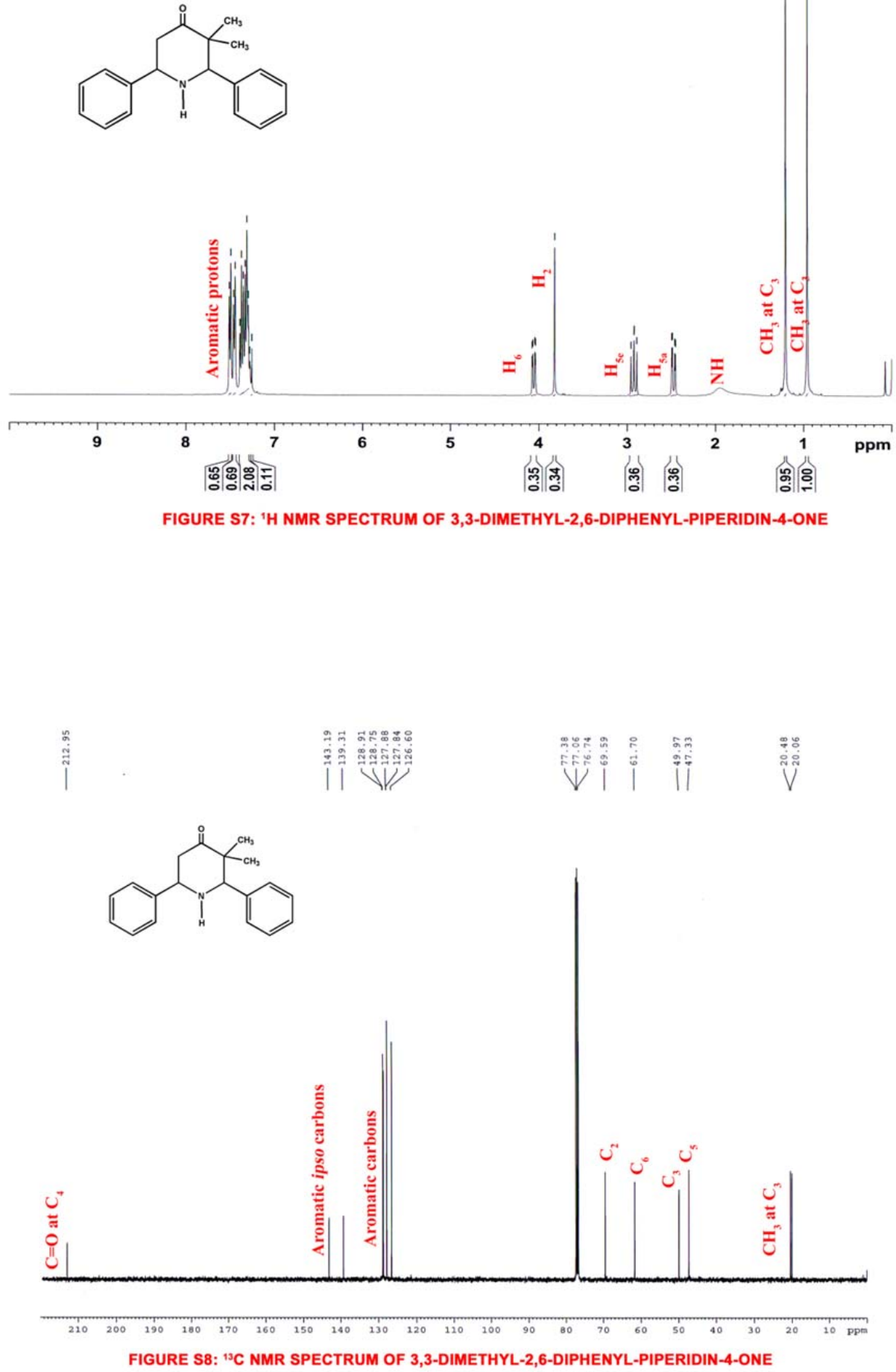


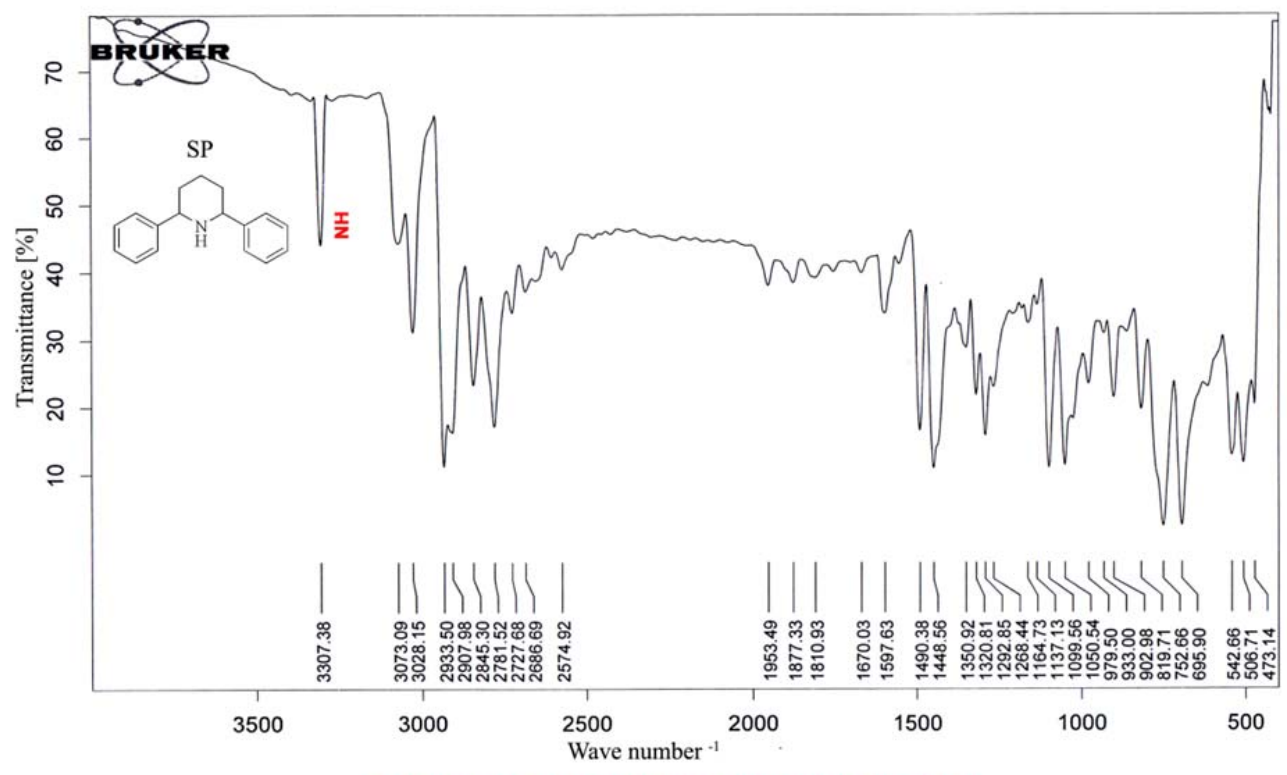

FIGURE S9: IR SPECTRUM OF 2,6-DIPHENYL-PIPERIDINE

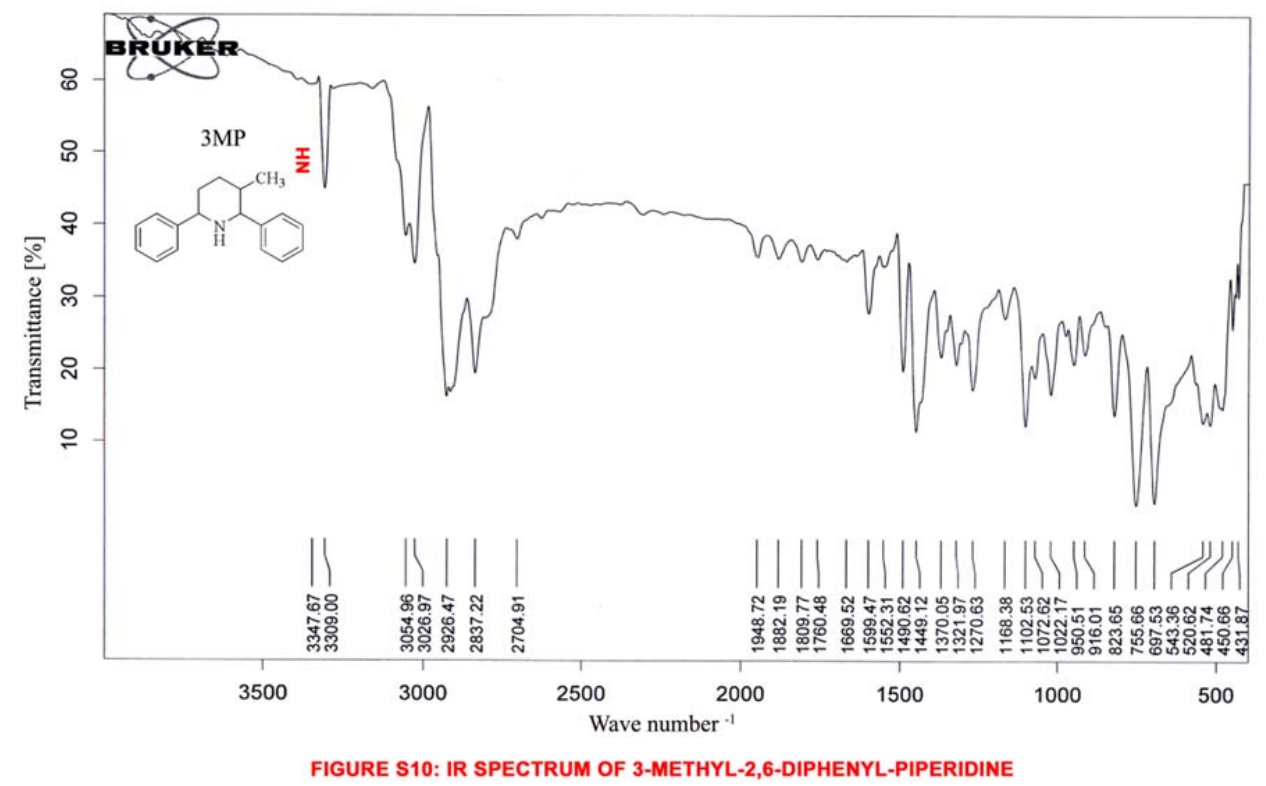




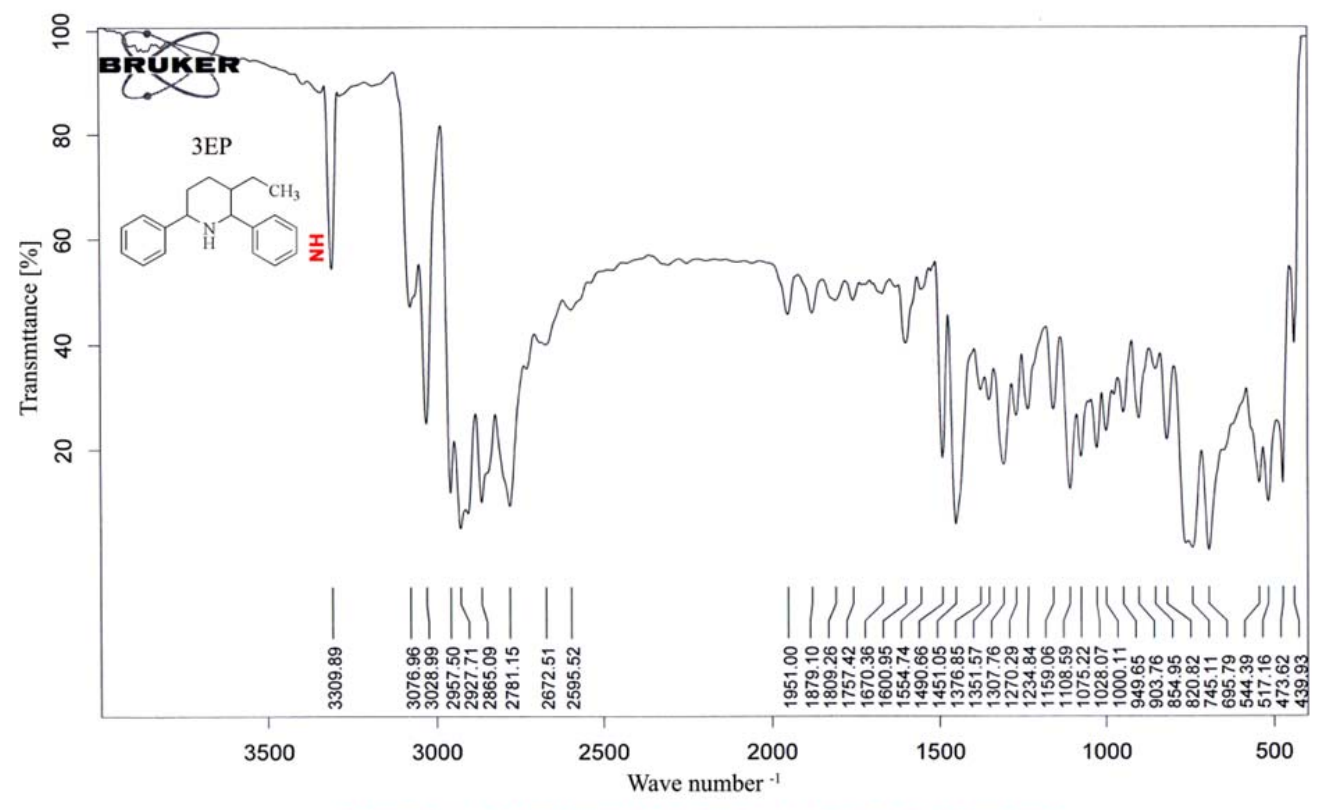

FIGURE S11: IR SPECTRUM OF 3-ETHYL-2,6-DIPHENYL-PIPERIDINE

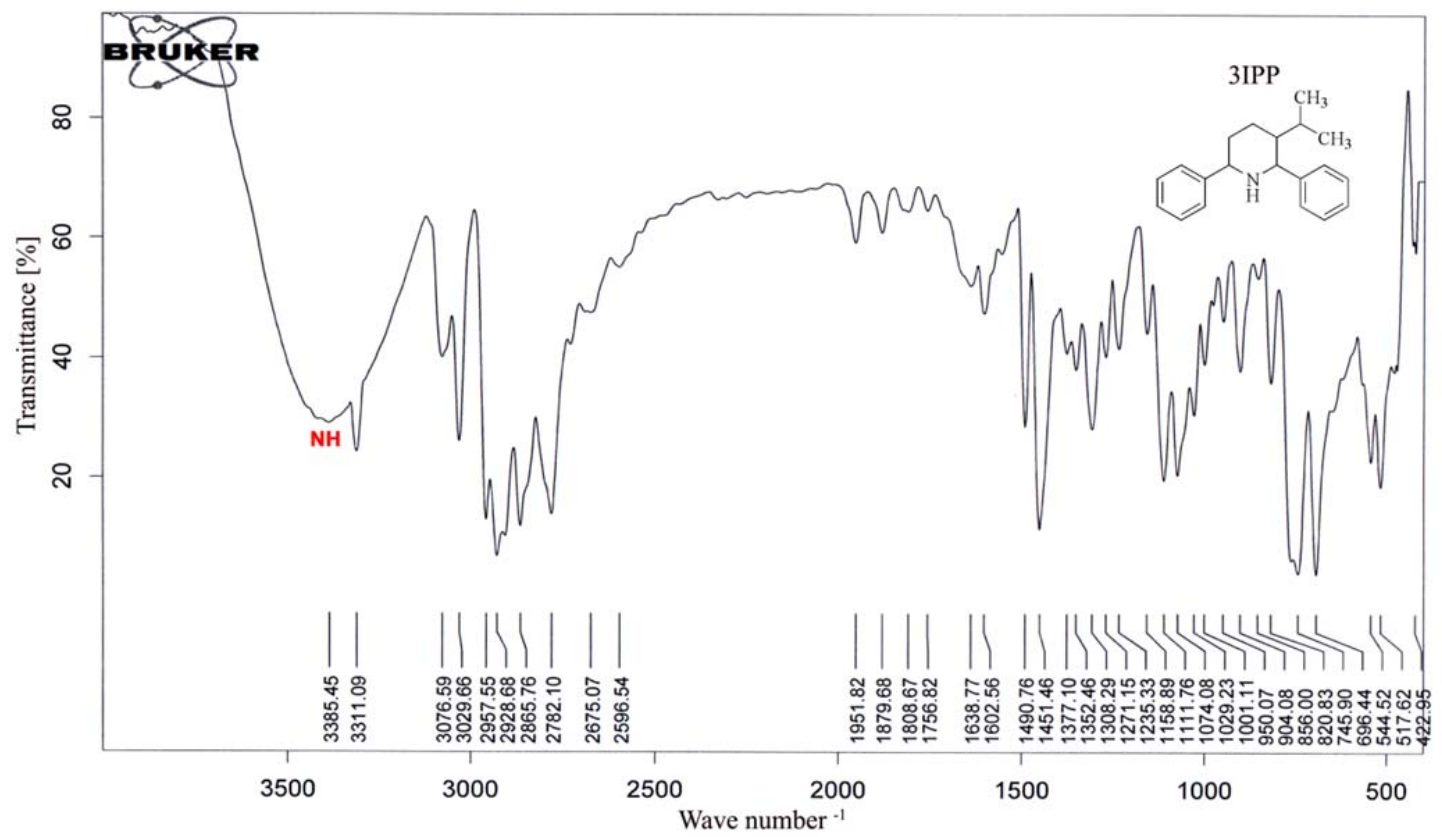

FIGURE S12: IR SPECTRUM OF 3-ISOPROPYL-2,6-DIPHENYL-PIPERIDINE 


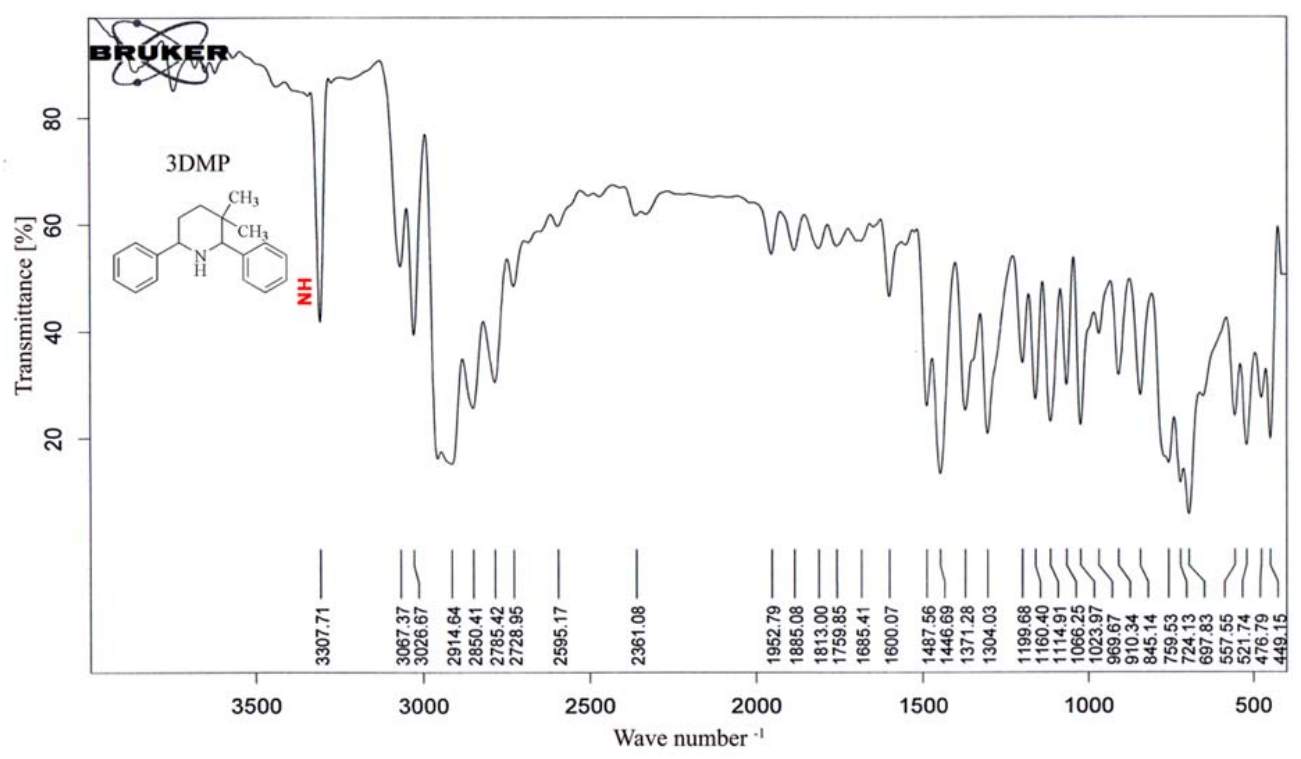

FIGURE S13: IR SPECTRUM OF 3,3-DIMETHYL-2,6-DIPHENYL-PIPERIDINE

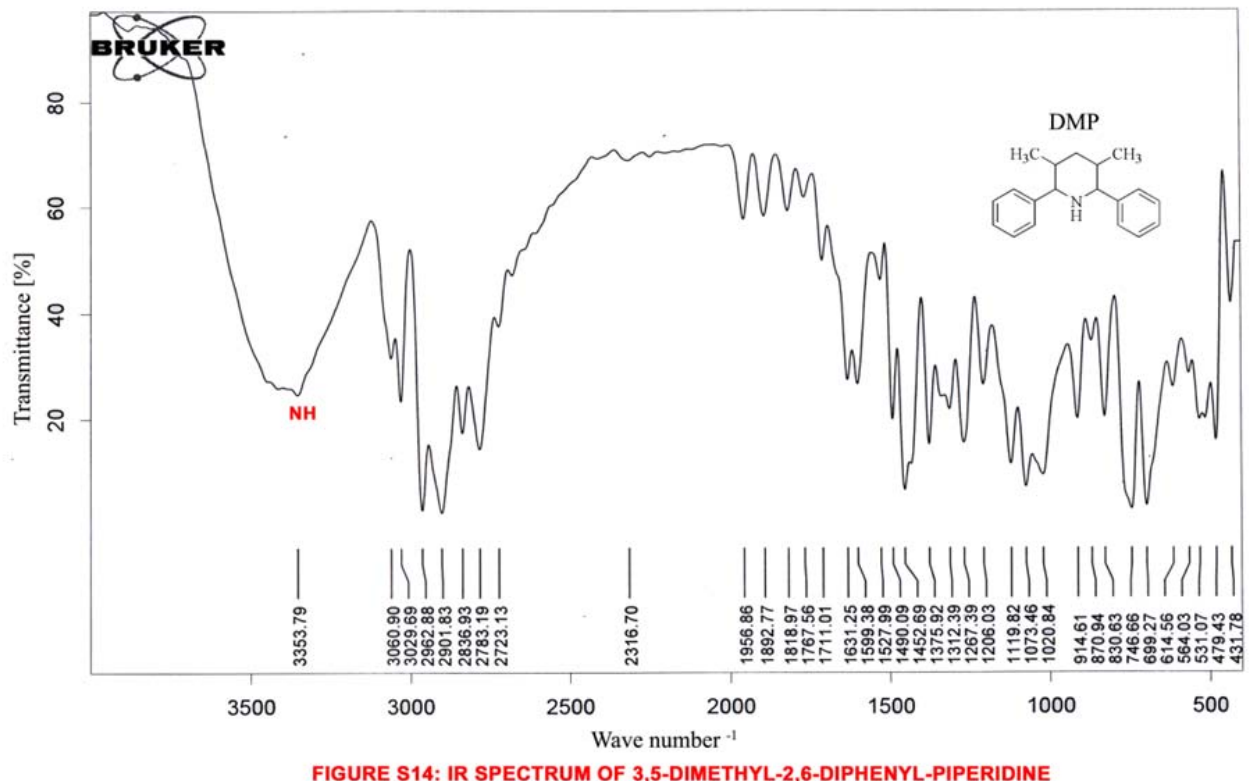



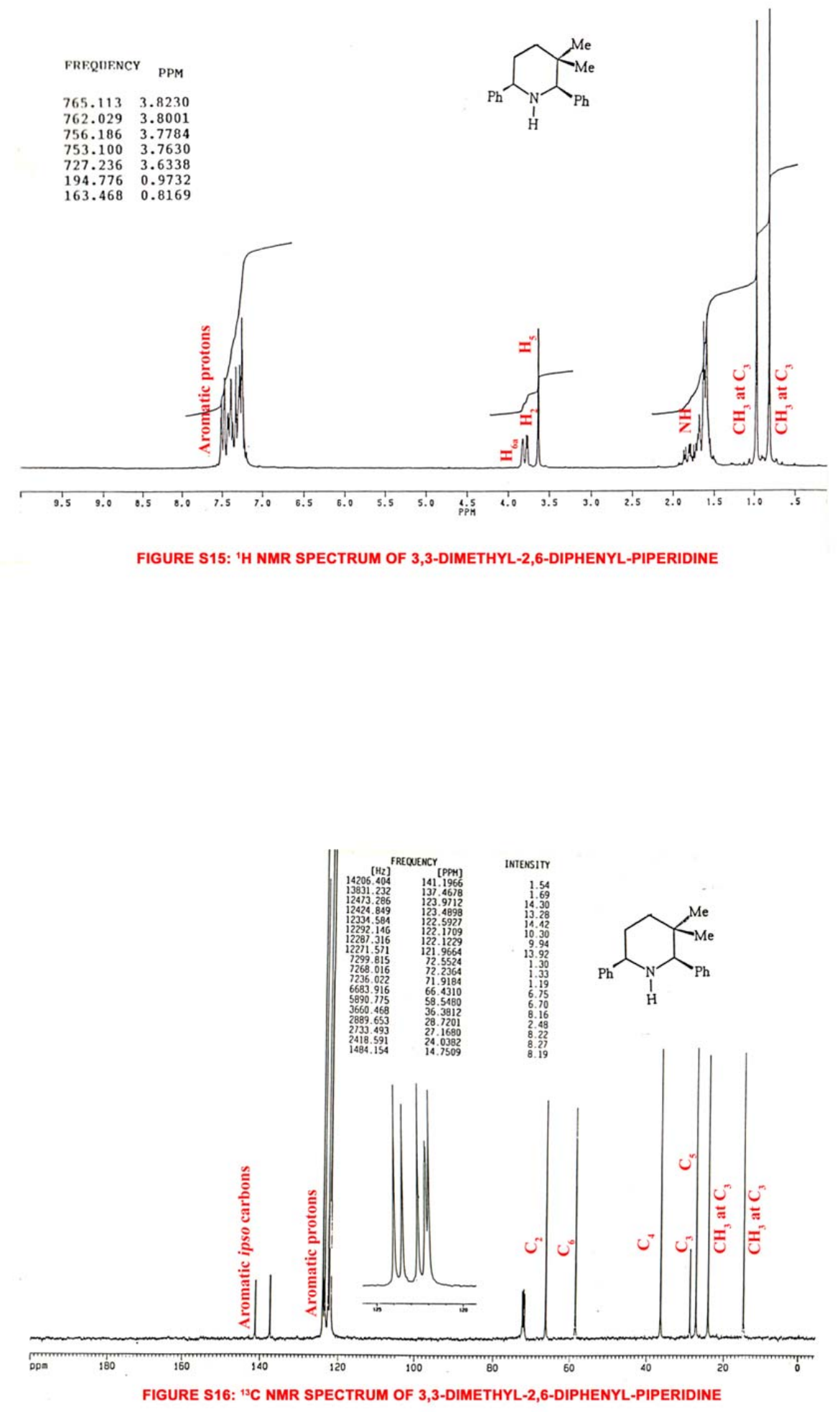




FIGURE S17: IR SPECTRUM OF 3-METHYL-2,7-DIPHENYL-1,4-DIAZEPAN-5-ONE

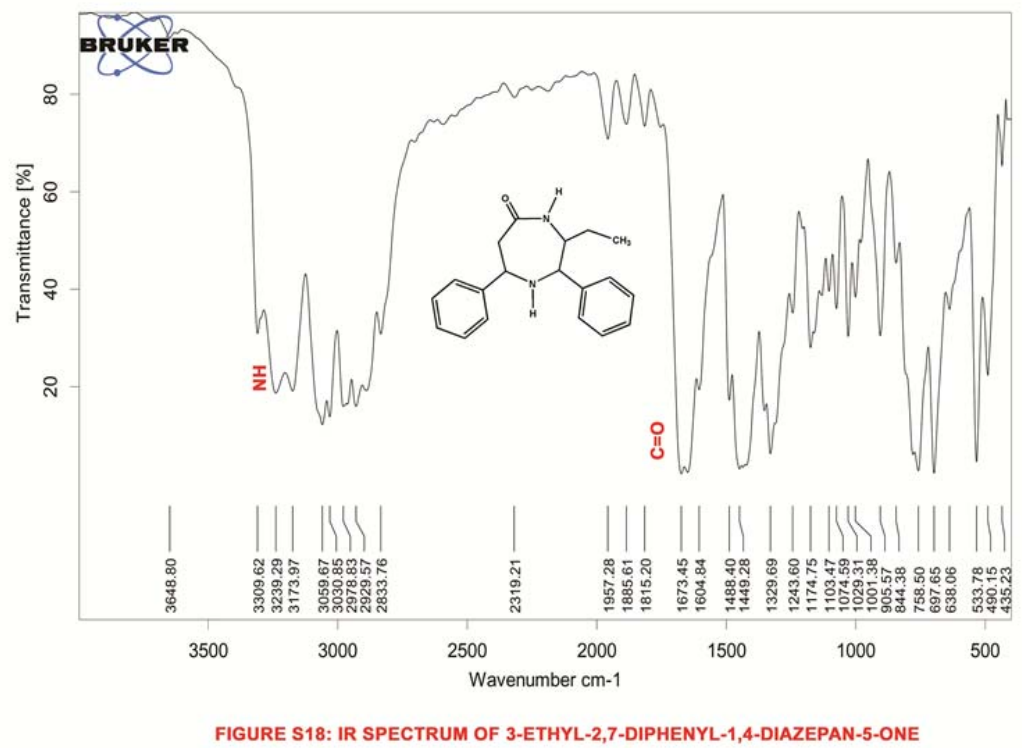






FIGURE S19: IR SPECTRUM OF 3-ISOPROPYL-2,7-DIPHENYL-1,4-DIAZEPAN-5-ONE

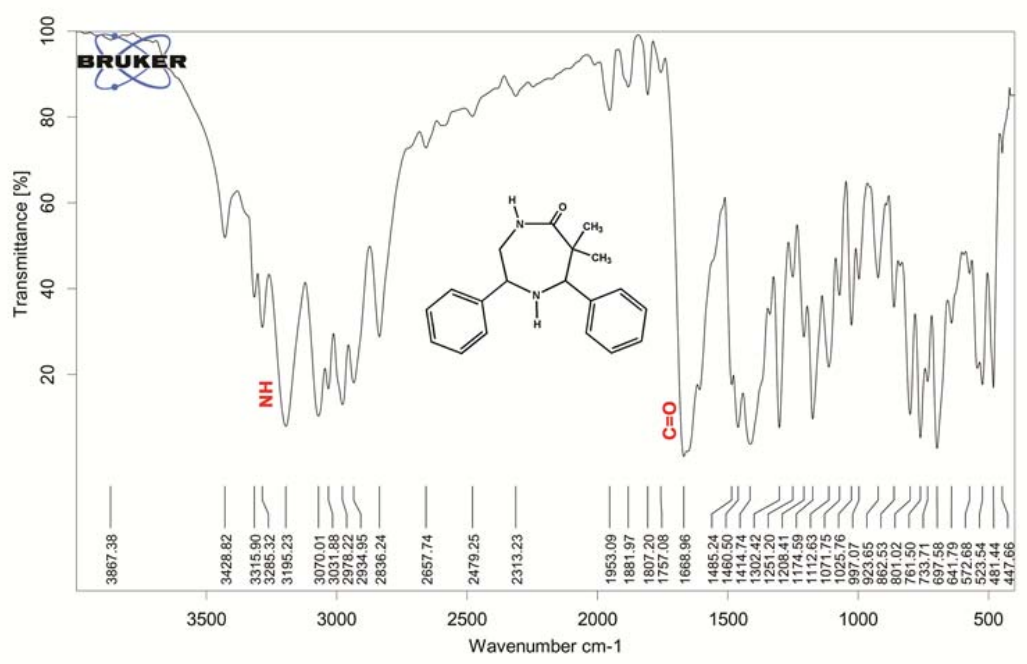

FIGURE S20: IR SPECTRUM OF 3,3-DIMETHYL-2,7-DIPHENYL-1,4-DIAZEPAN-5-ONE 


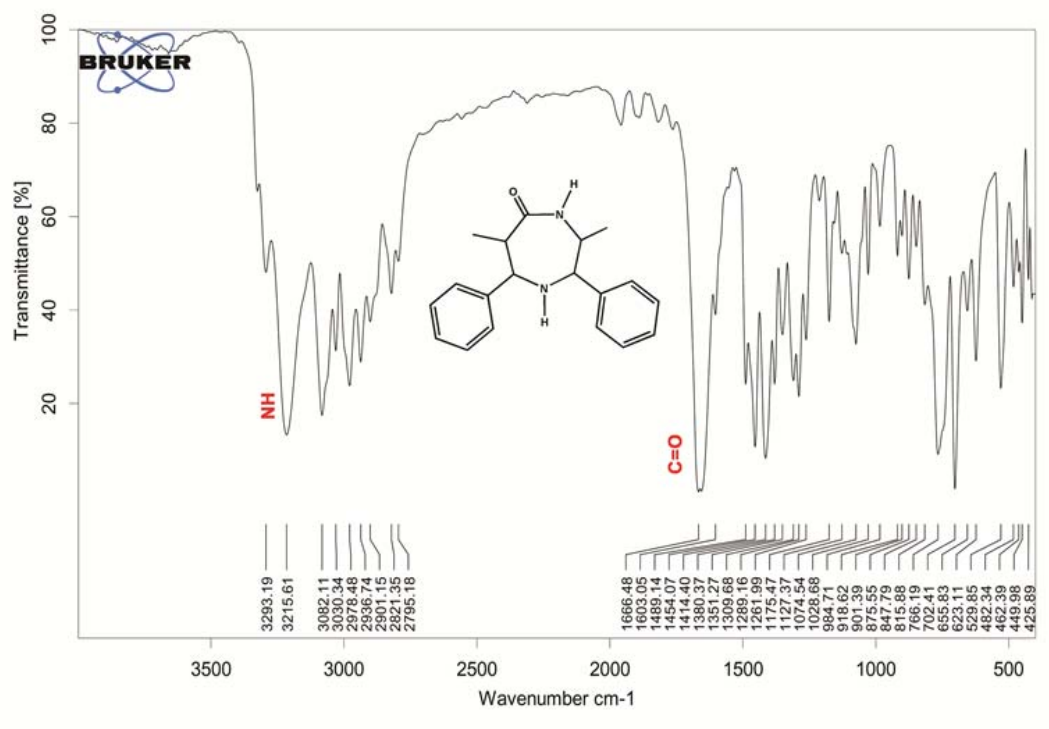

FIGURE S21: IR SPECTRUM OF 3,5-DIMETHYL-2,7-DIPHENYL-1,4-DIAZEPAN-5-ONE

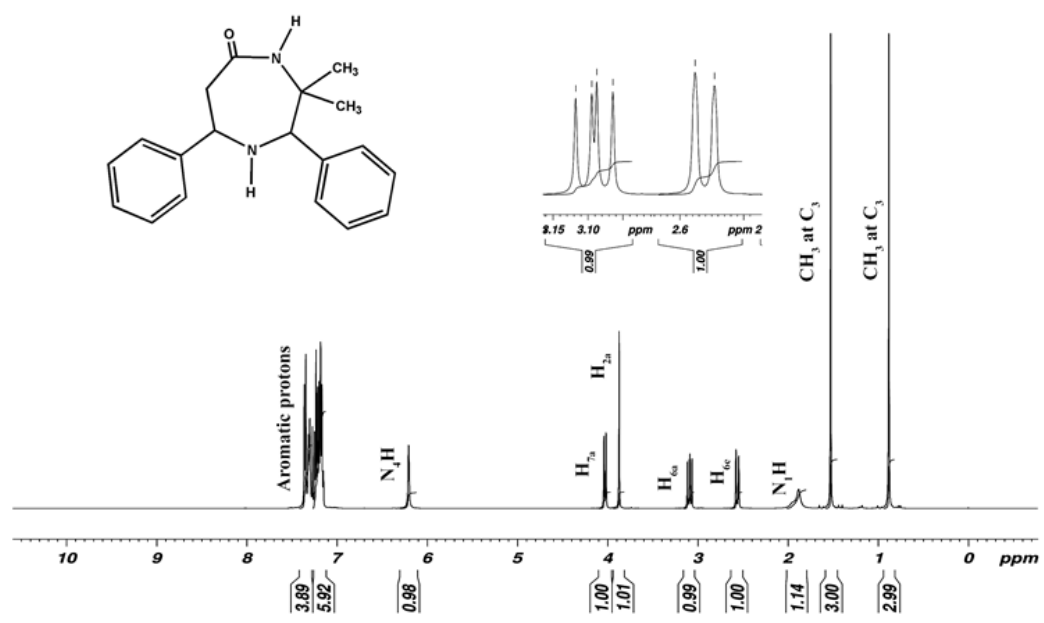

FIGURE S22: 'H NMR SPECTRUM OF 3,3-DIMETHYL-2,7-DIPHENYL-1,4-DIAZEPAN-5-ONE 


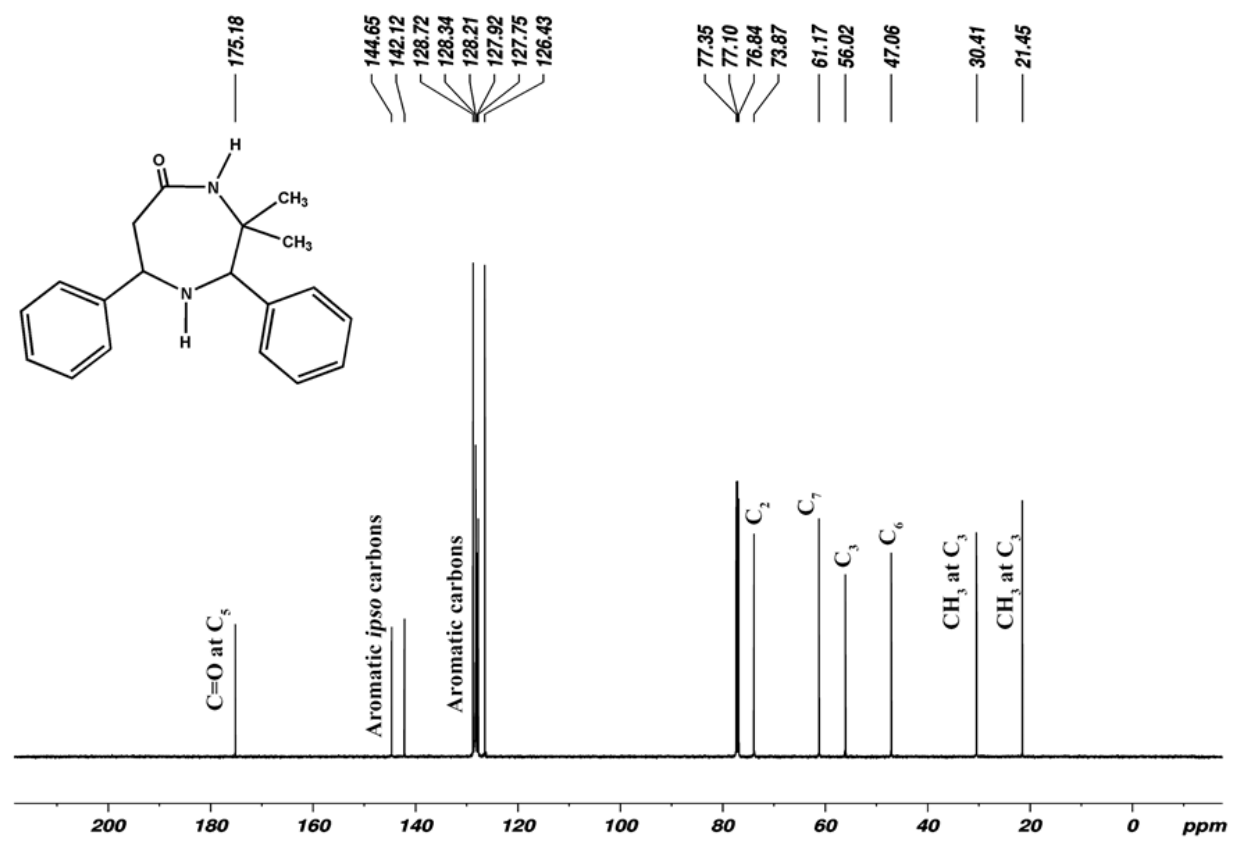

FIGURE S23: ${ }^{13} \mathrm{C}$ NMR SPECTRUM OF 3,3-DIMETHYL-2,7-DIPHENYL-1,4-DIAZEPAN-5-ONE 


\section{Table S1}

\begin{tabular}{|c|c|c|c|c|c|c|}
\hline Cpd No. & $\mathbf{R}^{1}$ & $\mathbf{R}^{2}$ & $\mathbf{R}^{3}$ & Yield (\%) & m.p $\left({ }^{\circ} \mathrm{C}\right)$ & Lit m.p $\left({ }^{\circ} \mathrm{C}\right)$ \\
\hline 1 & $\mathrm{CH}_{3}$ & $\mathrm{CH}_{3}$ & $\mathrm{H}$ & 50 & $210-211$ & $209-210^{[9]}$ \\
\hline 9 & $\mathrm{H}$ & $\mathrm{H}$ & $\mathrm{H}$ & 70 & $170-171$ & $171-172^{[5]}$ \\
\hline 10 & $\mathrm{CH}_{3}$ & $\mathrm{H}$ & $\mathrm{H}$ & 54 & $182-183$ & $184-185^{[5]}$ \\
\hline 11 & $\mathrm{CH}_{2} \mathrm{CH}_{3}$ & $\mathrm{H}$ & $\mathrm{H}$ & 80 & 198-199 & $200-201^{[5]}$ \\
\hline 12 & $i-\operatorname{Pr}$ & $\mathrm{H}$ & $\mathrm{H}$ & 90 & $185-186$ & $188-189^{[5]}$ \\
\hline 13 & $\mathrm{CH}_{3}$ & $\mathrm{H}$ & $\mathrm{CH}_{3}$ & 52 & $179-180$ & $180-181^{[5]}$ \\
\hline 14 & $\mathrm{H}$ & $\mathrm{H}$ & $\mathrm{H}$ & 50 & $101-102$ & $102-103^{[6]}$ \\
\hline 15 & $\mathrm{CH}_{3}$ & $\mathrm{H}$ & $\mathrm{H}$ & 62 & $86-87$ & $87^{[6]}$ \\
\hline 16 & $\mathrm{CH}_{2} \mathrm{CH}_{3}$ & $\mathrm{H}$ & $\mathrm{H}$ & 52 & $98-99$ & $90-92^{[6]}$ \\
\hline 17 & $i-\operatorname{Pr}$ & $\mathrm{H}$ & $\mathrm{H}$ & 50 & $124-125$ & $127^{[6]}$ \\
\hline 18 & $\mathrm{CH}_{3}$ & $\mathrm{CH}_{3}$ & $\mathrm{H}$ & 75 & $114-115$ & $114-115^{[8]}$ \\
\hline 19 & $\mathrm{CH}_{3}$ & $\mathrm{H}$ & $\mathrm{CH}_{3}$ & 80 & $130-131$ & $132-133^{[8]}$ \\
\hline 26 & $\mathrm{H}$ & $\mathrm{H}$ & $\mathrm{H}$ & 60 & $69-70$ & $69-71^{[6]}$ \\
\hline 27 & $\mathrm{CH}_{3}$ & $\mathrm{H}$ & $\mathrm{H}$ & 78 & $70-71$ & $71-72^{[6]}$ \\
\hline 28 & $\mathrm{CH}_{2} \mathrm{CH}_{3}$ & $\mathrm{H}$ & $\mathrm{H}$ & 80 & $52-53$ & $54^{[10]}$ \\
\hline 29 & $i-\operatorname{Pr}$ & $\mathrm{H}$ & $\mathrm{H}$ & 62 & $70-71$ & $71-72^{[10]}$ \\
\hline 30 & $\mathrm{CH}_{3}$ & $\mathrm{CH}_{3}$ & $\mathrm{H}$ & 74 & $94-95$ & $95-96^{[7]}$ \\
\hline 31 & $\mathrm{CH}_{3}$ & $\mathrm{H}$ & $\mathrm{CH}_{3}$ & 80 & $56-57$ & $55-56^{[6]}$ \\
\hline 37 & $\mathrm{H}$ & $\mathrm{H}$ & $\mathrm{H}$ & 68 & $170-171$ & $171-172^{[5]}$ \\
\hline 38 & $\mathrm{CH}_{3}$ & $\mathrm{H}$ & $\mathrm{H}$ & 52 & $182-183$ & $184-185^{[5]}$ \\
\hline 39 & $\mathrm{CH}_{2} \mathrm{CH}_{3}$ & $\mathrm{H}$ & $\mathrm{H}$ & 88 & 198-199 & $200-201^{[5]}$ \\
\hline 40 & $i-\operatorname{Pr}$ & $\mathrm{H}$ & $\mathrm{H}$ & 90 & $185-186$ & $188-189^{[5]}$ \\
\hline 41 & $\mathrm{CH}_{3}$ & $\mathrm{H}$ & $\mathrm{CH}_{3}$ & 50 & $179-180$ & $180-181^{[5]}$ \\
\hline
\end{tabular}


Table - S2

\begin{tabular}{|c|c|c|c|c|c|c|c|c|c|c|}
\hline Compound & $\mathrm{H}_{2}$ & $\mathrm{H}_{5 \mathrm{a}}$ & $\mathrm{H}_{5 \mathrm{e}}$ & $\mathrm{H}_{6 \mathbf{a}}$ & $\mathrm{H}_{6 \mathrm{e}}$ & $\mathrm{H}_{7 \mathrm{a}}$ & amine $\mathrm{NH}$ & aromatic & $\mathrm{CH}_{3}$ & CONH \\
\hline $\begin{array}{c}18 \\
\text { (3DMPO) }\end{array}$ & 3.84 & 2.48 & 2.93 & 4.05 & -- & -- & 1.95 & $7.25-7.50$ & $0.96,1.20$ & -- \\
\hline $\begin{array}{c}24 \\
\text { (3DMP) }\end{array}$ & 3.80 & 3.78 & 3.76 & 3.82 & -- & -- & 1.6 & 7.1-7.6 & $0.82,0.97$ & -- \\
\hline $\begin{array}{c}1 \\
\text { (3DMHD) }\end{array}$ & 3.87 & -- & -- & 3.09 & 2.56 & 4.03 & 1.88 & $7.17-7.36$ & $0.88,1.53$ & 6.21 \\
\hline
\end{tabular}


Table - S3

\begin{tabular}{|c|c|c|c|c|c|c|c|c|c|}
\hline Compound & $\mathrm{C} 6$ & C3 & $\mathrm{C7}$ & C5 & $\mathrm{C2}$ & ipso & $C=0$ & aromatic & $\mathrm{CH}_{3}$ at $\mathrm{C}_{3}$ \\
\hline $\begin{array}{c}18 \\
\text { (3DMPO) }\end{array}$ & 61.7 & 49.9 & -- & 47.3 & 69.6 & $143.1,139.3$ & 212.9 & 128.9-126.6 & $20.4,20.0$ \\
\hline $\begin{array}{c}24 \\
\text { (3DMP) }\end{array}$ & 58.5 & 28.7 & -- & 27.1 & 66.4 & $141.2,137.5$ & -- & $123.9-121.9$ & $14.7,24.0$ \\
\hline $\begin{array}{c}1 \\
\text { (3DMHD) }\end{array}$ & 47.1 & 56.0 & 61.2 & -- & 73.9 & $144.7,142.1$ & 175.2 & $128.7-126.4$ & $30.4,21.5$ \\
\hline
\end{tabular}

